MEMORANDUM

RM-4176-1-PR

MARCH 1966

\title{
THE (s-1, s) INVENTORY POLICY UNDER COMPOUND POISSON DEMAND: A THEORY OF RECOVERABLE ITEM STOCKAGE
}

G. J. Feeney and C. C. Sherbrooke

PREPARED FOR:

UNITED STATES AIR FORCE PROJECT RAND 


\section{MEMORANDUM}

RM-4176-1-PR

MARCH 1966

\section{THE (s-1, s) INVENTORY POLICY UNDER COMPOUND POISSON DEMAND: A THEORY OF RECOVERABLE ITEM STOCKAGE}

G. J. Feeney and C. C. Sherbrooke

This research is sponsored by the United States Air Force under Project RAND-Contract No. AF 49(638)-1700-monitored by the Directorate of Operational Requirements and Development Plans, Deputy Chief of Staff, Research and Development, Hq USAF. Views or conclusions contained in this Memorandum should not be interpreted as representing the official opinion or policy of the United States Air Force.

DISTRIBUTION STATEMENT

Distribution of this document is unlimited. 
, 


\section{PREFACE}

This Memorandum provides mathematical foundation for a new approach to setting stock levels on recoverable items. The study was initiated in response to a request by Logistics Plans, Headquarters USAF, and represents close cooperation and participation between representatives of RAND and the Air Force.

The Memorandum should be of particular interest to personnel involved in the details of implementation. Those interested primarily in the philosophy of the study and comparisons with the Air Force techniques should refer to G. J. Feeney, J. W. Petersen, and C. C. Sherbrooke, An Aggregate Base Stockage Policy for Recoverable Spare Parts, The RAND Corporation, RM-3644-PR, June 1963; and to G. J. Feeney and C. C. Sherbrooke, Systems Analysis and Supp1y Management, The RAND Corporation, RM-4054-PR, Apri1 1964.

This is an expanded version of $R M-4176-P R$ originally issued in September 1964. 

This Memorandum derives the simple analytic solution to the special, but important inventory problem in which the optimal policy is to reorder a unit whenever one is demanded. The demand distribution can be any compound Poisson; the resupplyidistribution is arbitrary. Both the backorder case and the lost sales case are solved by generalizing a queueing theorem due to $\mathrm{Pa} 1 \mathrm{~m}$.

The procedure is to calculate by item the steady state probabilities for the number of units in resupply (or repair). These probabilities completely describe the item's long term behavior, and are simply the normalized values of the compound Poisson demand distribution based on the mean of the resupply distribution but not on the distribution itself. Knowledge of these state probabilities enables us to compute several measures of item supply performance as a function of the spare stock, s. Traditional inventory analysis can then be applied to minimize total cost based on estimates of holding cost and supply performance cost.

The appendices contain a description of the algorithm and the computer program for calculating stuttering Poisson state probabilities and the measures of effectiveness for the backorder case. Numerical illustrations are also provided. 



\section{ACKNOWLEDGMENTS}

Several people have helped to stimulate our research. The efforts of George Fishman to derive analytic solutions for the recoverable spares problem were an early and important influence. Gabriele Michels programmed a simulation that supported our conjecture of Theorem 2 for the lost sales case. The subsequent proof of this theorem in Appendix $A$ owes much to the enthusiasm and advice of Herbert Scarf. Harrison Campbell has also been a constant source of encouragement. One of his comments led us to investigate and find analytic solutions for the measures of supply performance associated with delays. 


\section{'}




\section{CONTENTS}

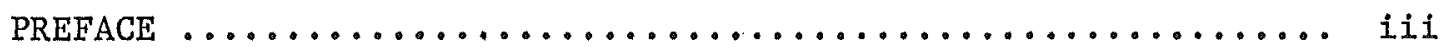

SUMMARY $\ldots \ldots \ldots \ldots \ldots \ldots \ldots \ldots \ldots \ldots \ldots \ldots \ldots \ldots \ldots \ldots \ldots \ldots \ldots \ldots \ldots \ldots \ldots$

ACKNOWLEDGMENTS $\ldots \ldots \ldots \ldots \ldots \ldots \ldots \ldots \ldots \ldots \ldots \ldots \ldots \ldots \ldots \ldots \ldots \ldots$

Section

I. INTRODUCTION $\ldots \ldots \ldots \ldots \ldots \ldots \ldots \ldots \ldots \ldots \ldots \ldots \ldots \ldots \ldots \ldots \ldots \ldots$

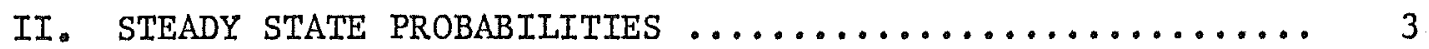

Inventory Theory - Poisson Demand .............. 3

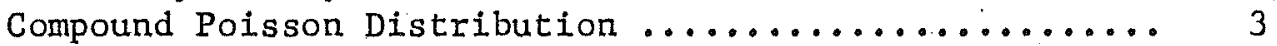

Inventory Theory - Compound Poisson Demand ......... 6

III. MEASURES OF SUPPLY PERFORMANCE ................ 9

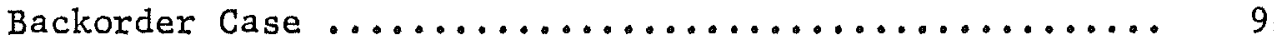

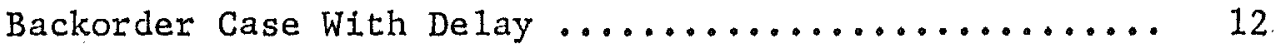

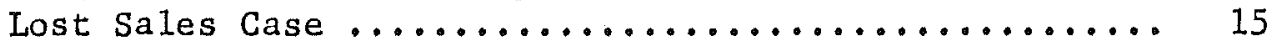

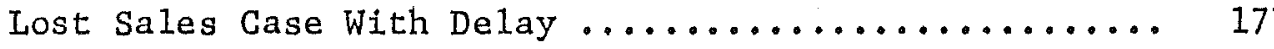

Comparison of the Backorder and Lost Sales Cases ...... 18

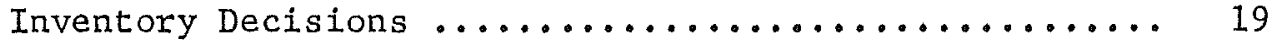

Appendix

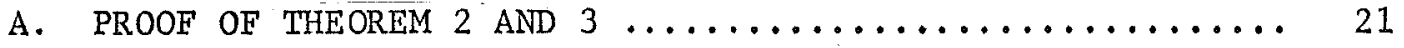

Proof of Theorem 2 for the Backorder Case ........... 21

Proof of Theorem 2 for the General Case ........... 21

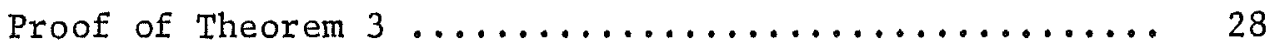

B. COMPUTATION OF STUTTERING POISSON DENSITY FUNCTION AND MEASURES OF PERFORMANCE IN THE BACKORDER CASE ..... 31

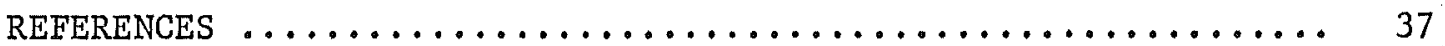




\section{INTRODUCTION}

Inventory policy is concerned, ultimately, with two decisions: when to reorder and how much to reorder. Much of the complexity of inventory analysis springs from the interaction of these two decisions. But consider those items whose cost is so high and/or whose demand is so 1 low that the optimal policy is to place a reorder ${ }^{*}$ immediately whenever a demand occurs. This is an important class of items including, for example, most aircraft recoverable spare parts that may cost hundreds of dollars and are typically demanded only a few times a year at an individual air base. The importance of this class is underlined by the Air Force investment in these recoverable spare parts which amounted to 6.9 billion dollars at the end of 1961. (1)

Inventory performance under this policy is determined entirely by a single decision, the spare stock, $s,{ }^{*}$ provided as protection against stockouts. If resupply were instantaneous, $s$ should be zero, but with positive resupply times the optimal $s$ is usually positive. This is a continuous review $(s-1, s)$. inventory policy which means that whenever a demand for an arbitrary number of units is accepted, a reorder is placed immediately for that number of units. This restores the total of stock on hand plus on order minus backorders to the spare stock level, s.

Let $\mathrm{x}(\mathrm{t})$ be a random variable representing the number of units in resupply at some arbitrary time, t. Throughout the Memorandum we shall distinguish between the backorder case in which $\mathrm{x}$ has the range $0 \leq x<\infty$, and the lost sales case in which $x$ is constrained to the range $0 \leq x \leq s$. In the lost sales case, any demand that occurs when there are $s$ units in resupply is rejected, because there is no stock on hand.

${ }^{*}$ Although the discussion is in terms of resupply, the analysis covers the repair case as well. More generally we can think of an item response distribution which is the composite of several distributions.

椋

The Air Force has called this quantity the total supply level, the stock control level, and most recently the requisitioning objective. 
Our objective will be to compute the steady state probability distribution measured at a random point of time for the number of units in resupply, $x$, since it is the only random variable when the inventory policy is $(s-1, s)$. For instance, when the quantity $s-x$ is positive it represents the stock on hand; when negative it gives the backorders. Thus the steady state probability distributions for stock on hand and backorders are obtained from the steady state distribution for the number of units in resupply by simply translating the origin of the distribution $s$ units.

These simplifications for the $(s-1, s)$ policy make it fairly easy to analyze the supply performance of an item under very general conditions. Specifically, a remarkable queueing theorem due to $\mathrm{Pa}_{\mathrm{m}}^{(2)}$ states that if demand is Poisson then the number of units in resupply in the steady state, $x$, is also Poisson for any distribution of resupply. The Poisson state probability depends on the mean of the resupply distribution, but not on the resupply distribution itself.

Several authors have considered this simplified inventory policy. Hadley and Whitin (3) have provided a thorough treatment of item theory for both the backorder case and the lost sales case for any resupply distribution under the assumption that demand has a Poisson distribution. Galliher, Morse, and Simond (4) generalized the demand assumption to the stuttering Poisson for the backorder case, but they considered only two types of resupply distribution -- constant and exponential.

In this Memorandum. we show that Palm's theorem can be generalized to any compound Poisson distribution (stuttering Poisson is a special. case). The number of units in resupply in the steady state is of the same compound Poisson form, again depending only on the mean of the arbitrary resupply distribution, enabling us to solve the backorder case and lost sales case completely. The compound Poisson model is far richer than the simple Poisson, but the calculations are still straightforward. 


\section{STEADY STATE PROBABILITIES}

\section{INVENTORY THEORY -- POISSON DEMAND}

Now we can restate Palm's well-known queueing theorem in the language of inventory control theory.

Theorem 1. Let $s$ be the spare stock for an item where demands are Poisson with rate $\lambda$ and the resupply time is an arbitrary probability distribution $\Psi(t)$ with mean $T$. In the backorder case, the steady state probabilities of $x$ units in resupply are given by the Poisson with rate $\lambda T$, i.e.

$$
\begin{aligned}
h(x)= & \text { steady state probability that } \\
& x \text { units are in resupply } \\
= & \frac{(\lambda T)^{x} e^{-\lambda T}}{x !} \quad 0 \leq x<\infty
\end{aligned}
$$

In the lost sales case where a demand is accepted only when stock on hand, $s-x$, is positive, the steady state probabilities of $x$ units in resupply are given by the normalized values of the Poisson with rate $\lambda \mathrm{T}$

$$
h(x)=\frac{\frac{(\lambda T) e^{-\lambda T}}{x !}}{\sum_{w=0}^{S} \frac{(\lambda T)^{W} e^{-\lambda T}}{w !}} \quad 0 \leq x \leq s
$$

It is easy to obtain a proof of Eq. (1) valid only for the backorder case. $(3,5)$ A proof of Eq. (2) will also be valid for Eq. (1) by letting $s \rightarrow \infty$, but the finite $s$ proof is complicated because the state probabilities depend on $s$. The first such proof due to Palm ${ }^{(2)}$ entailed a very laborious argument, but recently Scarf ${ }^{(6)}$ obtained a far more elegant proof.

COMPOUND POISSON DISTRIBUTION

The compound Poisson distribution is the obvious generalization of the simple Poisson. Figure 1 shows a time sample from both 


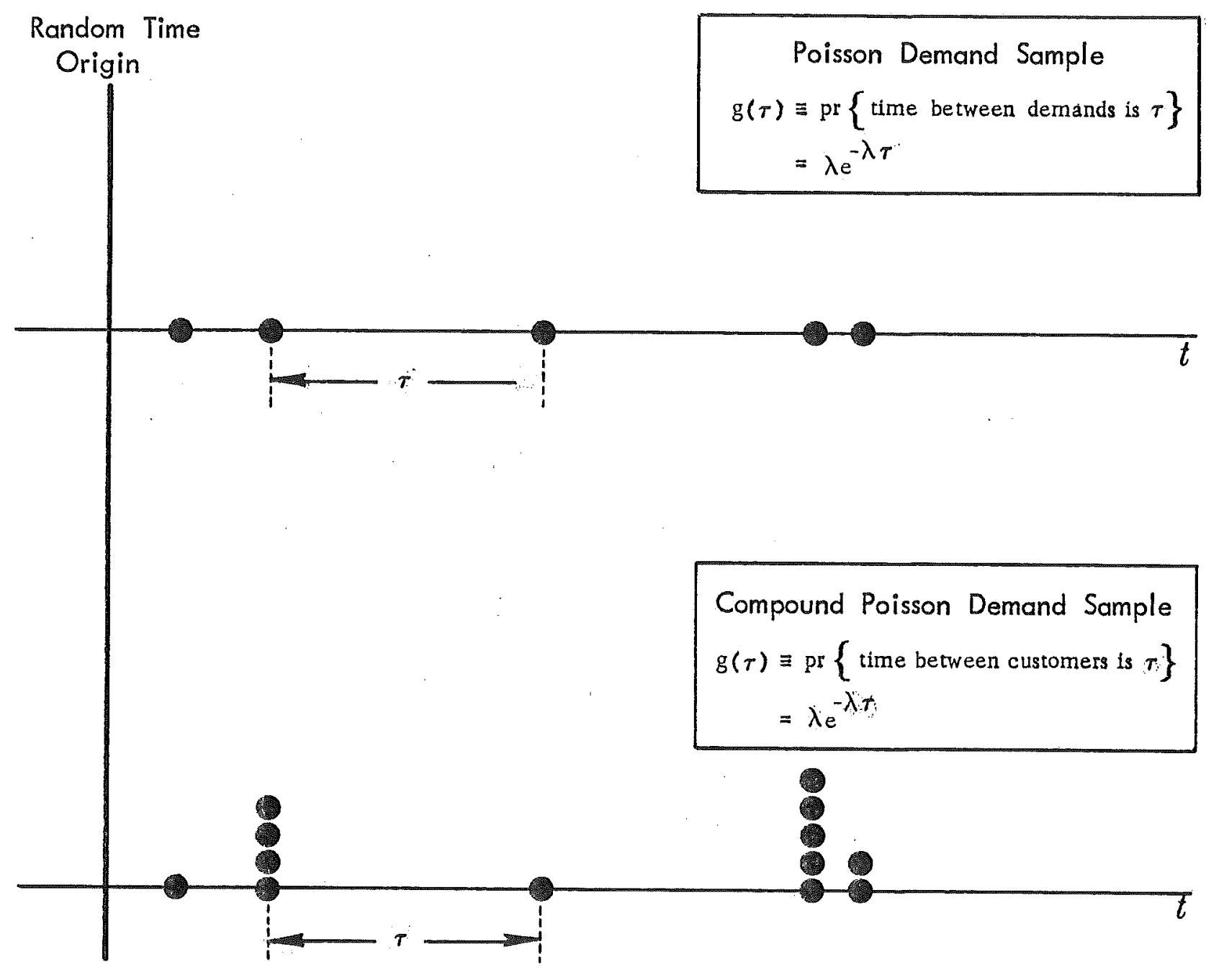

NOTE: Each represents a demand for one unit. In the Poisson case a customer always demands exactly one unit, but in the compound Poisson case multiple demands are possible.

Fig. 1 -- Comparison of Poisson and Compound Poisson Demand Sample 
distributions. Note that the compound Poisson has batches of demand rather than single demands, but the distribution of time is identical between batches for the compound Poisson and between demands for the simple Poisson. In both cases the time distribution is exponential. Conceptual1y, the compound Poisson can be visualized in an inventory problem as a series of customers with Poisson arrivals who demand an amount which has an independent discrete distribution, $f_{j}$.

The compounding distribution, $f_{j}$, may be any discrete distribution with any number of parameters. We shall assume the probability is zero that a customer places an order for zero demands, $f_{0}$, for any compound Poisson. This causes no loss of generality because any process with discrete distribution $f_{j}$ and positive $f_{0},\left(0<f_{0}<1\right)$, with customer arrival rate $\lambda$ is equivalent to the following process:

$$
f_{j}^{\prime}=\frac{f_{j}}{1-f_{0}} \quad j \geq 1
$$

$$
\begin{aligned}
& f_{0}^{\prime}=0 \\
& \lambda^{\prime}=\lambda\left(1-f_{0}\right)
\end{aligned}
$$

One particularly common choice of $f_{j}$ is the geometric, i.e.

$$
f_{j}=(1-\rho) \rho^{j-1} \quad j \geq 1, \quad 0 \leq \rho \leq 1 \text {. }
$$

In this case the compound Poisson distribution becomes the stuttering Poisson or geometric Poisson. For the purposes of our argument, however, there is no need to specialize $f_{j}$ at this time.

The compound Poisson has been described by many authors ${ }^{(7)}$ so only its most salient features will be mentioned.

1. Any compound Poisson distribution has a variance that exceeds or equals its mean. (When the variance equals the mean the compound Poisson reduces to the simple Poisson.)

2. The compound Poisson distributions are the most genera1 class of "memoryless" distributions, i.e., the number of demands occurring in a time 
period does not influence the probabilities of

demand in any other non-overlapping time period.

One of the reasons we are interested in generalizing the assumption of Poisson demand to distributions with a larger variance is that our demand data usually produces variances that exceed the means. ${ }^{*}$ Furthermore the physical model of customers who can order several units appears to be a reasonable description of many supply processes. $* x$

\section{INVENTORY THEORY - - COMPOUND POISSON DEMAND}

We shal1 now compute the probability of observing $x$ demands during some period of time when demand has a compound. Poisson distribution. Let the Poisson customer arrival rate during the time period be $\lambda$. Then there will be $x$ demands if there are $y \leq x$ customers and if the $y$ customers order a total of $x$ demands. The probability that $y$ customers order a total of $x$ demands is denoted by the $y$ fold convolution of $x, f^{y^{*}}(x)$. (7) Since the probability that there are $y$ customers and that the $y$ customers order a total of $x$ demands is

$$
\left(\left(\lambda^{y} e^{-\lambda}\right) / y^{\prime}\right) f^{y^{*}}(x) \quad 0 \leq x<\infty
$$

*Suggested are four possible explanations for the high variability observed at air bases for recoverable item demand.

1. Sympathetic replacement or undetected malfunctions -a maintenance man discovers a defective item on one aircraft and, as a result, inspects that item on other aircraft, replacing incipient failures.

2. Initial wearout -- some components like vacuum tubes may have a high probability of failure shortly after installation.

3. Damage during installation -- maintenance personnel may damage some parts like windshields during installation.

4. Flying programs are usually correlated between aircraft.

Feller ${ }^{(7)}$ describes two examples outside of inventory control theory where a compound Poisson model seems appropriate -- insurance and ecology. The queueing theorem would be applicable in these problems, too. 
the compound Poisson probability of $\mathrm{x}$ demands is

$$
p(x \mid \lambda)=\sum_{y=0}^{x} \frac{\lambda^{y} e^{-\lambda}}{y !} f^{y *}(x) \quad 0 \leq x<\infty
$$

Now we are ready to generalize Palm's theorem.

Theorem 2. Let s be the spare stock for an item where demands are compound Poisson with customer arrival rate $\lambda$ and the resupply time is an arbitrary distribution $\psi(t)$ with mean $T$. Assume that when a customer is accepted, a resupply time is drawn from $\Psi(t)$ that is applicable to all demands placed by that customer. In the backorder case, the steady state probabilities of $x$ units in resupply are given by the compound Poisson with rate $\lambda T$, i.e.

$$
h(x)=p(x \mid \lambda T) \quad 0 \leq x<\infty .
$$

In the lost sales case under the assumption that a customer is accepted only when the stock on hand, $s-x$, equals or exceeds the number of demands made by the customer, the steady state probabilities for the number of units in resupply are

$$
h(x)=\frac{p(x \mid \lambda T)}{\sum_{w=0}^{s} p(w \mid \lambda T)} \quad 0 \leq x \leq s .
$$

Since the proof is rather lengthy, it is deferred to Appendix A. Notice that in this thorem the ambiguity in queue discipline for multiple demands by a customer requires the addition of two conditions to Palm's theorem. In the lost sales case we specified that a customer order would be accepted or rejected "in toto." Alternatively, we could have allowed partial fills of an order until stock on hand is reduced to zero. This case is also solved in Appendix A, and yields a simple solution. 
The second assumption is that one resupply time is drawn from $\psi(t)$ for a customer's demands. This is an important, but quite resonable assumption. It means the resupply times for all demands a particular customer places are perfectly correlated.

The alternative assumption is that each demand has a resupply time drawn independently from $\psi(t)$. This seems far less appropriate for our physical model in which each customer may place an order for several units because it means that the resupply for a customer dribbles in one unit at a time. Since this assumption has been embodied in earlier papers, it is of interest to examine the effect of the assumption.

Let us define the total stock to be the stock on hand plus on order minus backorders. Then the alternative assumption yields an inventory policy which is $(R, Q)$ with $Q=1$ : when the total stock falls to the reorder level $R$ or below, a number $n$ of reorders of size $Q$ is placed sufficient to bring the total stock above $R$. Our assumption, by contrast, yields an $(s-1, s)$ policy: one reorder is placed when the total stock falls to $s-1$ or less and the size of the order is just sufficient to bring the total stock back to s. In the simple Poisson case where demands do not occur in bunches, the policies are, of course, identical.

The $Q=1$ resupply assumption was employed by Galliher, Morse, Simond. They computed the steady state probabilities for the backorder case with stuttering Poisson demand where the compounding distribution, $\left\{f_{j}\right\}$, is given by Eq. (4). With a constant resupply time they found the variance of the steady state probability distribution was $\lambda(1+p) /(1-p)^{2}$. Of course, this is a special case of our theorem. However, with an exponential resupply time they found the variance* of the steady state probability distribution was $\lambda /(1-p)^{2}$. Galliher, Morse and Simond found it surprising that a variable resupply time (exponential) decreased the variability in the system. We now realize that this phenomenon is due to the $Q=1$ assumption, since the distribution of resupply does not affect the state probabilities under our resupply assumption.

\footnotetext{
"In the original article this variance was given incorrectly. Using generating functions, it is easy to show that the variance in their notation should be $\rho /(1-\psi)$.
} 
III. MEASURES OF SUPPLY PERFORMANCE

\section{BACKORDER CASE}

Now that we have obtained the steady state occupancy probabilities in Eqs. (6) and (7) for the number of units in resupply, we can compute several measures of item supply performance. Each depends on the spare stock, s, for the item as well as the demand parameters for the compound Poisson and the mean resupply time, T. In the backorder case we define three measures.

$$
\begin{aligned}
R(s) \equiv & \begin{array}{l}
\text { Ready rate }-- \text { the probability that an item } \\
\text { observed at a random point in time has no } \\
\text { backorders. }
\end{array} \\
F(s) \equiv & \text { Fills - the expected number of demands per } \\
& \text { time period for an item that can be filled } \\
& \text { immediately from stock on hand. } \\
S(s) \equiv & \text { Units in service -- the expected number of } \\
& \text { units in routine resupply at a random point } \\
& \text { in time. }
\end{aligned}
$$

The measures are defined so that each increases with s. Ready rate is the simplest measurement distinguishing between two possibilities -- zero backorders or positive backorders. When ready rate is our performance criterion, it means that the number of backorders on an item is irrelevant.

On the other hand, we may be interested in the expected number of backorders incurred during some period of time. Instead of computing this directly we define a performance measurement that, like ready rate, increases with s. The appropriate measure for a time period is fills, since the expected number of backorders is equal to the expected number of demands minus the expected number of fills. The expected number of demands is, of course, independent of the spare stock.

Finally, we may want to consider the duration of the backorders as well as the number of backorders. At a random point in time the expected number of units in routine resupply is defined as the expected number of units in resupply minus the expected number of units in a backordered condition. For instance, the number of units in routine resupply equals the number of units in resupply when this number is less than or equal to $s$; it equals $s$ when the number of units in resupply exceeds $s$ since the excess demand is in a backordered condition. Since the period is of 
unit length due to the way we defined $\lambda$, the expected number of units in a backordered condition equals the expected number of backorder days during the period. The expected number of units in resupply is, of course, independent of the spare stock, s. Under this criterion one backorder lasting ten days has a penalty equal to ten backorders lasting one day each.

We shall demonstrate the calculation of these measures for the backorder case before discussing the lost sales case. Under the conditions of Theorem 2 , the steady state probability that $x$ units are in resupply in the backorder case is given by Eq. (6):

$$
h(x)=p(x \mid \lambda T)=\sum_{y=0}^{x} \frac{(\lambda T)^{y} e^{-\lambda T}}{y !} f^{y^{*}}(x)
$$

Then the ready rate, $R(s)$ is simply the steady state probability that $s$ or less units are in resupply:

$$
R(s)=\sum_{x=0}^{s} p(x \mid \lambda T)
$$

In order to calculate $F(s)$, we recall that the expected number of customers per time period is $\lambda$. The expected number of fills per customer is simply obtained from the steady state probabilities. There will always be one fill if $s-1$ or less units are in resupply since each customer places an order for at least one unit. There will be a second fill if $s-2$ or less units are in resupply and the customer order is for two or more demands. Continuing the argument we find that the expected number of fills per period is 


$$
\begin{aligned}
F(s)= & \lambda\left\{\sum_{x=0}^{s-1} p(x \mid \lambda T)+\left(1-f_{1}\right) \sum_{x=0}^{s-2} p(x \mid \lambda T)\right. \\
& +\left(1-f_{1}-f_{2}\right) \sum_{x=0}^{s-3} p(x \mid \lambda T) \ldots \cdot \\
& \left.+\left(1-f_{1}-f_{2} \cdot \ldots f_{s-1}\right) p(0 \mid \lambda T)\right\},
\end{aligned}
$$

where $f_{j}$ is the probability that a customer demands exactly $j$ units. Using Eq. (9) we can rewrite $F(s)$ as

(10)

$$
F(s)=\lambda\left\{R(s-1)+\left(1-f_{1}\right) R(s-2)+\left(1-f_{1}-f_{2}\right) R(s-3)\right.
$$

$$
\left.. .+\left(1-f_{1}-f_{2} \cdot . f_{s-1}\right) R(0)\right\}
$$

The expected number of demands can be obtained by letting $s \rightarrow \infty$, and it equals $\lambda \bar{f}$ where $\bar{f}$ is the average customer order size.

To calculate the number of units in routine resupply we observe that when the total number of units in resupply, $x$, is less than $s$, there will be $x$ units in routine resupply. When $x$ exceeds $s$, however, there will be exactly $s$ units in routine resupply. Thus the expected number of units in routine resupply at a random time is given by

$$
\begin{aligned}
S(s) & =\sum_{x=1}^{s} x p(x \mid \lambda T)+s \sum_{x=s+1}^{\infty} p(x \mid \lambda T) \\
& =\sum_{x=1}^{\infty} x p(x \mid \lambda T)-\sum_{x=s}^{\infty}(x-s) p(x \mid \lambda T) \\
& =\lambda T \bar{f}-\sum_{x=s}^{\infty}(x-s) p(x \mid \lambda T)
\end{aligned}
$$


The expected number of units in any resupply condition is $\lambda \bar{T} \bar{f}$. In fact, we could alternatively have written down Eq. (12) from the definition of $S(s)$ as the expected number of units in resupply minus the expected number of units in a backordered condition.

In Appendix B there is a description of an algorithm and computer program for calculating the steady state probabilities, ready rate, fills and services when the demand distribution is stuttering Poisson and demand is backordered. Table 1 displays some sample output from this program.

\section{BACKORDER CASE WITH DELAY}

Suppose that immediate response of the supply system is not required and a fill is redefined as a demand that can be met within a time $\tau$. As before, the demand initiates an immediate resupply action. This formulation includes the original definition of a fill as the special case when $\tau=0$. More generally, each measure of performance can be extended in this manner by computing the steady state probabilities that $x$ units are in resupply where each unit has been in the resupply process for at least $\tau$.

Theorem 3a. Let demand be compound Poisson with customer arrival rate $\lambda$ and the resupply time be an arbitrary distribution $\psi(t)$ with mean $T$. In the backorder case, the steady state probabilities of $x$ units in resupply each of which has been in the resupply process for at least $T$ are given by the compound Poisson with rate $\lambda \mathrm{T} \alpha$, i.e.

$$
h(x)=p(x \mid \lambda T \alpha)=\sum_{y=0}^{x} \frac{(\lambda T \alpha)^{y} e^{-\lambda T \alpha}}{y !} f^{y *}(x)
$$

where

$$
\alpha=1 / T \int_{T}^{\infty}[1-\Omega(t)] d t
$$

and 
Table 1

MEASURES OF SUPPLY PERFORMANCE UNDER STUTTERING POISSON DEMAND-BACKORDER CASE

\begin{tabular}{|c|c|c|c|c|c|c|c|c|c|c|c|c|}
\hline $\mathbf{s}$ & $p(s)$ & $R(s)$ & $F(s)$ & $s(s)$ & $p(s)$ & $R(s)$ & $F(s)$ & $s(s)$ & $p(s)$ & $R(s)$ & $F(s)$ & $s(s)$ \\
\hline & \multicolumn{2}{|c|}{$x=.25$} & \multicolumn{2}{|c|}{$V=.25$} & \multicolumn{2}{|c|}{$x=.50$} & \multicolumn{2}{|c|}{$V=.50$} & \multicolumn{2}{|c|}{$x=1.00$} & \multicolumn{2}{|c|}{$v=1.00$} \\
\hline $\begin{array}{l}0 \\
1 \\
2 \\
3 \\
4 \\
5 \\
6 \\
7 \\
8\end{array}$ & $\begin{array}{l}.77880 \\
.19470 \\
.02434 \\
.00203 \\
.00013 \\
.00001\end{array}$ & $\begin{array}{r}.77880 \\
.97350 \\
.99784 \\
.99987 \\
.99999 \\
1.00000\end{array}$ & $\begin{array}{l}.0000 \\
.2947 \\
.2434 \\
.2495 \\
.2500 \\
.2500\end{array}$ & $\begin{array}{l}.0000 \\
.2212 \\
.2477 \\
.2499 \\
.2500 \\
.2500\end{array}$ & $\begin{array}{l}.60653 \\
.30327 \\
.07582 \\
.01264 \\
.00158 \\
.00016 \\
.00001\end{array}$ & $\begin{array}{l}.60653 \\
.90980 \\
.98561 \\
.99825 \\
.99983 \\
.99999 \\
1.00000\end{array}$ & $\begin{array}{l}.0000 \\
.3033 \\
.4549 \\
.4928 \\
.4992 \\
.4999 \\
.5000\end{array}$ & $\begin{array}{l}.0000 \\
.3935 \\
.4837 \\
.4981 \\
.4998 \\
.5000 \\
.5000\end{array}$ & $\begin{array}{l}.36788 \\
.36788 \\
.18394 \\
.06131 \\
.01533 \\
.00307 \\
.00051 \\
.00007 \\
.00001\end{array}$ & $\begin{array}{r}.36788 \\
.73576 \\
.91970 \\
.98101 \\
.99634 \\
.99941 \\
.99992 \\
.99999 \\
1.00000\end{array}$ & $\begin{array}{r}.0000 \\
.3679 \\
.7358 \\
.9197 \\
.9810 \\
.9963 \\
.9994 \\
.9999 \\
1.0000\end{array}$ & $\begin{array}{l}.0000 \\
.6321 \\
.8964 \\
.9767 \\
.9957 \\
.9993 \\
.9999 \\
1.0000 \\
1.0000\end{array}$ \\
\hline & \multicolumn{2}{|c|}{$x=.25$} & \multicolumn{2}{|c|}{$V=.50$} & \multicolumn{2}{|c|}{$x=.50$} & \multicolumn{2}{|c|}{$V=1.00$} & \multicolumn{2}{|c|}{$x=1.00$} & \multicolumn{2}{|c|}{$V=2.00$} \\
\hline $\begin{array}{l}0 \\
1 \\
2 \\
3 \\
4 \\
5 \\
6 \\
7 \\
8 \\
9 \\
10 \\
11 \\
12\end{array}$ & $\begin{array}{l}.04648 \\
.09405 \\
.03658 \\
.01413 \\
.00542 \\
.00207 \\
.00079 \\
.00030 \\
.00011 \\
.00004 \\
.00002 \\
.00001\end{array}$ & $\begin{array}{l}.84648 \\
.94054 \\
.97721 \\
.99124 \\
.99666 \\
.99873 \\
.99952 \\
.99982 \\
.99993 \\
.99997 \\
.99999 \\
1.00000\end{array}$ & $\begin{array}{l}.0000 \\
.1411 \\
.2038 \\
.2308 \\
.2421 \\
.2468 \\
.2487 \\
.2495 \\
.2498 \\
.2499 \\
.2500 \\
.2500\end{array}$ & $\begin{array}{l}.0000 \\
.1535 \\
.2130 \\
.2359 \\
.2446 \\
.2480 \\
.2492 \\
.2497 \\
.2499 \\
.2500 \\
.2500 \\
.2500\end{array}$ & $\begin{array}{l}.71653 \\
.15923 \\
.07077 \\
.03080 \\
.01318 \\
.00556 \\
.00232 \\
.00096 \\
.00039 \\
.00016 \\
.00006 \\
.00003 \\
.00001\end{array}$ & $\begin{array}{l}.71653 \\
.87576 \\
.94653 \\
.97733 \\
.99050 \\
.99607 \\
.99838 \\
.99934 \\
.99973 \\
.99989 \\
.99996 \\
.99998 \\
.99999\end{array}$ & $\begin{array}{l}.0000 \\
.2388 \\
.3715 \\
.4394 \\
.4722 \\
.4876 \\
.4945 \\
.4976 \\
.4990 \\
.4996 \\
.4998 \\
.4999 \\
.5000\end{array}$ & $\begin{array}{l}.0000 \\
.2835 \\
.4077 \\
.4612 \\
.4839 \\
.4933 \\
.4973 \\
.4989 \\
.4996 \\
.4998 \\
.4999 \\
.5000 \\
.5000\end{array}$ & $\begin{array}{l}.51342 \\
.22819 \\
.12677 \\
.06667 \\
.03370 \\
.01652 \\
.00791 \\
.00371 \\
.00171 \\
.00078 \\
.00035 \\
.00016 \\
.00007 \\
.00003 \\
.00001 \\
.00001\end{array}$ & \begin{tabular}{|}
.51342 \\
.74160 \\
.86837 \\
.93504 \\
.96874 \\
.98527 \\
.99318 \\
.99689 \\
.99860 \\
.99938 \\
.99972 \\
.99988 \\
.99995 \\
.99998 \\
.99999 \\
1.00000
\end{tabular} & $\begin{array}{r}.0000 \\
.3423 \\
.6085 \\
.7817 \\
.8839 \\
.9405 \\
.9703 \\
.9856 \\
.9931 \\
.9968 \\
.9985 \\
.9993 \\
.9997 \\
.9999 \\
.9999 \\
1.0000\end{array}$ & $\begin{array}{r}.0000 \\
.4866 \\
.7450 \\
.8766 \\
.9416 \\
.9728 \\
.9876 \\
.9944 \\
.9975 \\
.9989 \\
.9995 \\
.9998 \\
.9999 \\
1.0000 \\
1.0000 \\
1.0000\end{array}$ \\
\hline & \multicolumn{2}{|c|}{$x=.25$} & \multicolumn{2}{|c|}{$\mathrm{V}=.75$} & \multicolumn{2}{|c|}{$x=.50$} & \multicolumn{2}{|c|}{$V=1.50$} & \multicolumn{2}{|c|}{$x=1.00$} & \multicolumn{2}{|c|}{$v=3.00$} \\
\hline $\begin{array}{r}0 \\
1 \\
2 \\
3 \\
4 \\
5 \\
6 \\
7 \\
8 \\
9 \\
10 \\
11 \\
12 \\
13 \\
14 \\
15 \\
16 \\
17 \\
18 \\
19\end{array}$ & \begin{tabular}{|l|}
.08250 \\
.05516 \\
.02930 \\
.01555 \\
.00824 \\
.00436 \\
.00231 \\
.00122 \\
.00064 \\
.00034 \\
.00018 \\
.00009 \\
.00005 \\
.00003 \\
.00001 \\
.00001
\end{tabular} & $\begin{array}{l}.88250 \\
.93765 \\
.96695 \\
.98250 \\
.99074 \\
.99511 \\
.99742 \\
.99864 \\
.99928 \\
.99962 \\
.99980 \\
.99990 \\
.99994 \\
.99997 \\
.99998 \\
.99999\end{array}$ & $\begin{array}{l}.0000 \\
.1103 \\
.2724 \\
.2071 \\
.2263 \\
.2370 \\
.2429 \\
.2461 \\
.2479 \\
.2489 \\
.2494 \\
.2497 \\
.2498 \\
.2499 \\
.2499 \\
.2500\end{array}$ & $\begin{array}{l}.0000 \\
.1175 \\
.1799 \\
.2129 \\
.2304 \\
.2396 \\
.2445 \\
.2471 \\
.2485 \\
.2492 \\
.2496 \\
.2498 \\
.2499 \\
.2499 \\
.2500 \\
.2500\end{array}$ & \begin{tabular}{|l|}
.77880 \\
.09735 \\
.05476 \\
.03068 \\
.01712 \\
.00952 \\
.00528 \\
.00292 \\
.00161 \\
.00089 \\
.00049 \\
.00027 \\
.00015 \\
.00008 \\
.00004 \\
.00002 \\
.00001 \\
.00001
\end{tabular} & $\begin{array}{l}.77880 \\
.87615 \\
.93091 \\
.96159 \\
.97871 \\
.98823 \\
.99351 \\
.99643 \\
.99804 \\
.99893 \\
.99941 \\
.99968 \\
.99983 \\
.99991 \\
.99995 \\
.99997 \\
.99998 \\
.99999\end{array}$ & $\begin{array}{l}.0000 \\
.1947 \\
.3164 \\
.3909 \\
.4359 \\
.4626 \\
.4784 \\
.4876 \\
.4929 \\
.4960 \\
.4977 \\
.4987 \\
.4993 \\
.4996 \\
.4998 \\
.4999 \\
.4999 \\
.5000\end{array}$ & $\begin{array}{l}.0000 \\
.2212 \\
.3450 \\
.4141 \\
.4526 \\
.4738 \\
.4856 \\
.4921 \\
.4957 \\
.4976 \\
.4987 \\
.4993 \\
.4996 \\
.4998 \\
.4999 \\
.4999 \\
.5000 \\
.5000\end{array}$ & $\begin{array}{l}.60653 \\
.15163 \\
.09477 \\
.05844 \\
.03564 \\
.02153 \\
.01290 \\
.00767 \\
.00453 \\
.00266 \\
.00156 \\
.00091 \\
.00053 \\
.00030 \\
.00017 \\
.00010 \\
.00006 \\
.00003 \\
.00002 \\
.00001 \\
.00001\end{array}$ & $\begin{array}{l}.60653 \\
.75816 \\
.85293 \\
.91138 \\
.94701 \\
.96854 \\
.98143 \\
.98910 \\
.99364 \\
.99630 \\
.99786 \\
.99876 \\
.99929 \\
.99959 \\
.99977 \\
.99987 \\
.99992 \\
.99996 \\
.99998 \\
.99999 \\
.99999\end{array}$ & $\begin{array}{l}.0000 \\
.3033 \\
.5307 \\
.6918 \\
.8016 \\
.8743 \\
.9214 \\
.9514 \\
.9703 \\
.9820 \\
.9891 \\
.9935 \\
.9961 \\
.9977 \\
.9987 \\
.9992 \\
.9995 \\
.9997 \\
.9998 \\
.9999 \\
.9999\end{array}$ & $\begin{array}{l}.0000 \\
.3935 \\
.6353 \\
.7824 \\
.8710 \\
.9240 \\
.9554 \\
.9740 \\
.9849 \\
.9913 \\
.9950 \\
.9971 \\
.9983 \\
.9991 \\
.9995 \\
.9997 \\
.9998 \\
.9999 \\
.9999 \\
1.0000 \\
1.0000\end{array}$ \\
\hline
\end{tabular}

NOTE: $X$ equals the mean. $V$ equeis the variance.

${ }^{a}$ See Appendix 2 for derivations of these functions under stuttering Poisson demand. 


$$
\Omega(t)=\int_{0}^{t} \psi(\xi) d \xi
$$

Note that in this case the value of $\alpha,(0 \leq \alpha \leq 1)$, depends on the form of the resupply distribution. The proof, given in the Appendix, is a direct consequence of Takacs (5) result for Poisson input extended to compound Poisson input.

The measures of performance are easy to compute. We define $\mathrm{R}_{T}(\mathrm{~s})$ as the probability that an item observed at a random point in time has no backorders that have lasted $T$ or longer. Then we obtain

$$
\mathrm{R}_{\tau}(\mathrm{s})=\sum_{\mathrm{x}=0}^{\mathrm{s}} \mathrm{p}(\mathrm{x} \mid \lambda \mathrm{T} \alpha)
$$

In computing $\mathrm{F}_{\tau}(\mathrm{s})$ we relate the customer arrival at a random instant to the steady state probability for the number of units still in the resupply process after a time period, $T$. If a customer arrives to find $y$ units in resupply, there will after a time period, $\tau$, still be $x$. Denoting the customer order size by $i$, we find for $x=0$ that there will be $i$ fills for $i \leq s$ and $s$ fills for $i>s$. In addition there is a probability $(1-\alpha)$ that the customer arrival itself will be resupplied during $\tau$ which means that in the case $i>s$ the remaining $i-s$ units will be filled. When $x=1$ and $i s s-1$ there will be $i$ fills; for $i>s-1$ there will be $s-1$ fills and with probability $(1-\alpha)$ an additional $i-(s-1)$ fills. The resulting equation is a generalization of Eq. (10),

$$
F_{\tau}(s)=\lambda\left\{p(0 \mid \lambda T \alpha)\left[\sum_{i=1}^{s} i f_{i}+s \sum_{i=s+1}^{\infty} f_{i}+(1-\alpha) \sum_{i=s+1}^{\infty}(i-s) f_{i}\right]\right.
$$

$$
+p(1 \mid \lambda T \alpha)\left[\sum_{i=1}^{s-1} \text { if }_{i}+(s-1) \sum_{i=s}^{\infty} f_{i}+\right.
$$




$$
\begin{aligned}
& \left.(1-\alpha) \sum_{i=s}^{\infty}(i-(s-1)) f_{i}\right] \\
& \left.\ldots+\left[\sum_{x=s}^{\infty} p(x \mid \lambda T \alpha)\right]\left[(1-\alpha) \sum_{i=1}^{\infty} i f_{i}\right]\right\} .
\end{aligned}
$$

Despite the complicated appearance of Eq. (14a), a very simple recurrence relation can be obtained.

The expected number of units in routine resupply at a random point in time, $S_{\tau}(s)$, equals the expected number of units in resupply minus the expected number of units in a backordered condition. The expected number of units in resupply is still $\lambda \overrightarrow{T f}$, but the expected number of backordered units includes only those units on which the backorder has lasted $T$ or longer. Thus we obtain an expression similar to Eq. (12)

$$
S_{T F}(s)=\lambda T \bar{T}-\sum_{x=s}^{\infty}(x-s) p(x \mid \lambda T \alpha) .
$$

\section{LOST SALES CASE}

In the lost sales case, ready rate is a meaningless criterion. By definition, the probability is one that an item has no backorders. However, we can calculate the fills per time period, $F(s)$, and the units in service, $s(s)$. Assume the condition of theorem 2 that a customer is accepted only when the number of empty channels equals or exceeds the number of demands that customer makes. Then with spare stock, the steady state occupancy probabilities for the lost sales case are from Eq. (7):

$$
h(x)=\frac{p(x \mid \lambda T)}{\sum_{w=0}^{s} p(w \mid \lambda T)} \quad 0 \leq x \leq s
$$

where 


$$
p(x \mid \lambda T)=\sum_{y=0}^{x} \frac{(\lambda T)^{y} e^{-\lambda T}}{y !} f^{y *}(x)
$$

The steady state probabilities enable us to compute the expected number of fills per customer. There will be exactly one fill if there are s-1 or less units in resupply and the customer order is for exactly one. There will be exactly two fills if there are $s-2$ or less units in resupply and the customer order is for two, etc. Since the probability of a customer arrival in any time interval, $\Delta t$, is just $\lambda \Delta t$ independent of the state of the system, the expected number of fills per period in the lost sales case when customer orders are completely accepted or rejected, $F_{c}(s)$, is

$$
\begin{aligned}
& F_{c}(s)=\lambda\left\{f_{1} \sum_{x=0}^{s-1} h(x)+2 f_{2} \sum_{x=0}^{s-2} h(x) \cdots \cdot+s f_{s} h(0)\right\} \\
& F_{c}(s)=\frac{\lambda}{\sum_{w=0}^{s} p(w \mid \lambda T)}\left\{f_{1} \sum_{x=0}^{s-1} p(x \mid \lambda T)+2 f_{2} \sum_{x=0}^{s-2} p(x \mid \lambda T) \cdots+s f_{s} p(0 \mid \lambda T)\right\} \\
& \text { (17) } F_{c}(s)=\frac{\lambda}{R(s)}\left\{f_{1} R(s-1)+2 f_{2} R(s-2) \ldots+s f_{s} R(0)\right\}
\end{aligned}
$$

For the partial fill rule, the steady state probabilities are derived in Appendix $A$ in equations (A.11) and (A.12). They are

$$
\begin{array}{rlr}
h(x) & =p(x \mid \lambda T) / \Delta(s) & 0 \leq x<s \\
h(s) & =p(s \mid \lambda T) \sum_{i=s}^{\infty} f^{* *}(i) / \Delta(s) & s \\
& =\left(\sum_{y=0}^{s}\left((\lambda T) e^{-\lambda T / y !}\right) \sum_{i=s}^{\infty} f^{y^{*}}(i)\right) / \Delta(s)
\end{array}
$$


where

$$
\Delta(s)=R(s-1)+\sum_{y=0}^{s}\left((\lambda T)^{y} e^{-\lambda T} / y !\right) \sum_{i=s}^{\infty} f^{y^{*}}(i)
$$

We can derive $F_{p}(s)$, the expected number of fills per period under that partial fill rule, by the argument used for $F(s)$ in Eq. (10). The only difference is that the probabilities are normalized by $\Delta s$,

$$
F_{p}(s)=\frac{F(s)}{\Delta(s)}
$$

The expected number of units in service under the complete fill rule, $S_{c}(s)$, and under the partial fill rule, $S_{p}(s)$, are easily computed from the state probabilities analogous to Eq. (11):

$$
\begin{gathered}
S_{c}(s)=\frac{1}{R(s)} \sum_{x=1}^{s} x_{p}(x \mid \lambda T) ; \\
S_{p}(s)=\frac{1}{\Delta(s)}\left\{\sum_{x=1}^{s-1} x p(x \mid \lambda T)+s \sum_{y=0}^{s} \frac{(\lambda T)^{y} e^{-\lambda T}}{y !} \sum_{i=s}^{\infty} f^{* *}(i)\right\} ;
\end{gathered}
$$

\section{LOST SALES CASE WITH DELAY}

It is possible to compute measures of performance after a delay for the lost sales case. In this case, however, the probabilities are not simple modifications of those without delay as in Eq. (13) for the backorder case. For this reason the appropriate steady state probabilities derived in Theorem 3 of Appendix A are not displayed here. The procedure for computing the measures of performance is then straightforward though tedious. 
COMPARISON OF THE BACKORDER AND LOST SALES CASES

In this section we shall derive inequality relationships among the three fill measurements and among the three service measurements* for any finite value of the spare stock and arbitrary compound Poisson distributions. This will bring the structural properties of the measures into perspective.

Under the lost sales case the partial acceptance of a customer order can only increase the fills and services over the numbers obtained under the complete acceptance rule. Therefore, for any spare stock, s, and arbitrary compound Poisson demand,

$$
\begin{aligned}
& F_{p}(s) \geq F_{c}(s), \\
& S_{p}(s) \geq S_{c}(s) .
\end{aligned}
$$

A strict inequality is not possible because the acceptance rules become identical when the arbitrary compound Poisson is chosen to be the simple Poisson.

Now we sha11 relate the lost sales cases to the backorder case. Since $\Delta(s)<1$ for any finite $s$, Eq. (17) indicates

$$
F_{p}(s)>F(s)
$$

Let us compare $S(s)$ with $S_{p}(s)$. Under the partial fill rule there is a positive probability that some demand will be lost for any finite s and arbitrary compound Poisson demand. Since this demand is accepted in the backorder case we can establish a strict inequality and generalize Eq. (21b) to

$$
\mathrm{S}(\mathrm{s})>\mathrm{S}_{\mathrm{p}}(\mathrm{s}) \geq \mathrm{S}_{\mathrm{c}}(\mathrm{s})
$$

\footnotetext{
"The measures of supply performance in the backorder case with delay will not be discussed here because of their obvious relationship to the backorder case without delay.
} 
It is impossible to establish a general relationship between $F(s)$ and $F_{c}(s)$. The following argument makes this clear. In the Poisson case, the partial fill rule and the complete fill rule for the lost sales case become identical. Thus for the simple Poisson we find from Eq. (22)

$$
F_{p}(s)=F_{c}(s)>F(s)
$$

When demand is compound Poisson, however, it is possible to select a compounding distribution, $f_{j}$, such that $F_{c}(s)=0$ and $F(s)$ is positive. This can be accomplished by selecting

$$
\begin{aligned}
& \mathrm{f}_{s+1}=1 ; \\
& \mathrm{f}_{j}=0 \quad j \neq s+1 . .
\end{aligned}
$$

Thus we have shown that no general relationship between $F_{c}(s)$ and $F(s)$ can be established for al1 compound Poisson distributions.

\section{INVENTORY DECISIONS}

We have calculated a variety of steady state performance measures for compound Poisson demand under an $(s-1, s)$ inventory policy. Traditional inventory analysis can now be applied to determine optimal spare stock by item. Let $G(s)$ represent an arbitrary performance measurement (ready rate, fills, or units in service for either the backorder case or the lost sales case). Let $k_{1}$ be the per unit value of performance, $k_{2}$ be the holding cost in per cent for some specified time period (obsolescence, warehousing; interest), and $c$ be the unit cost. Using a digital computer, the objective is to maximize

$$
H(s)=k_{1} G(s)-k_{2} c s .
$$


In general, there may be more than one relative maximum because $G(s)$ is not necessarily convex, but the absolute maximum is easy to find. The admissible values of $s$ are discrete, and for low demand $i$ tems the optimal value of $s$ is not large. 
Appendix A

PROOF OF THEOREM 2 AND 3

\section{PROOF OF THEOREM 2 FOR THE BACKORDER CASE}

Before proving the theorem we present a simple proof based, on Palm's theorem and valid only for the backorder case.

- In a compound Poisson demand process the customer arrival process is simple Poisson. Therefore, Palm's theorem in Eq. (1) gives the steady state probability that $y$ customers are in the system when demand is compound Poisson. Now the probability that $x$ demands are in resupply in the steady state is simply the probability that there are $y$ customers in the steady state and these customers placed a total of $\mathrm{x}$ demands summed over all $\mathrm{y}$ with $\mathrm{y} \leq \mathrm{x}$. This is because of our resupply assumption: any customer who has not been resupplied has his entire order in the resupply process. Since the resupply process is independent of the customer order size, the probability that any $y$ customers place a total of $x$ demands is just $f^{y^{*}}(x)$.

PROOF OF THEOREM 2 FOR THE GENERAL CASE

The proof for the lost sales case cannot be obtained as a simple generalization of Poisson demand to compound Poisson demand as in the backorder case above, because the decision to accept or reject the next customer depends on the number of demands in the system, not the number of customers.

Therefore, we shall give a complete proof of this theorem, extending the argument employed by Scarf for Poisson demand. Like Scarf, we do not give a completely rigorous proof for all resupply distributions, but only for a subclass of distributions, which is, however, dense in the class of all distributions.

First we shall focus our attention on the individual customers and the resupply distribution, $\Psi(t)$. Let us assume that $\Psi(t)$ is a distribution for the recurrence time for a state of a continuous time, finite state Markov process. This means that when a reorder for a customer is placed, it passes through a number of states (not necessarily in sequence) prior to delivery, and that the length of time spent in each state is given by a negative exponential distribution.

Suppose the states of the Markov process are labeled $1,2, \ldots \mathrm{n}$, and $\psi(t)$ is the distribution of time for a customer to leave state 1 and then return to it. Let the transition probabilities be 
(A. 1)

$$
\mathrm{p}_{j k}(\Delta t)=\delta_{j k}+\mathrm{q}_{j k} \Delta t+o\left(\Delta t^{2}\right)
$$

for small $\Delta t$; where $\delta_{j k}$ is the Kronecker delta; $\delta_{j k}=1$ if $j=k$, $\delta_{j k}=0$ if $j \neq k$. In this formulation

$$
\begin{array}{ll}
q_{j k} \geq 0 & \text { if } j \neq k \\
q_{j k} \leq 0 & \text { if } j \neq k .
\end{array}
$$

From the definition of a continuous time Markov process and the meaning of a stationary probability that a customer is in state. $j, \pi_{j}$, we obtain the following equations:

$$
\sum_{j=1}^{N} \pi_{j} q_{j k}=0
$$

The resupply rate for the Markov process is simply obtained by weighting the transition rates from each state $j,(j \neq 1)$, to state 1 by the steady state probability of being in state $j$ :

$$
\sum_{j=2}^{N} \pi_{j} q_{j 1}=-\pi_{1} q_{11}=\frac{1}{T} \text {, }
$$

where $T$ is the average time for the distribution, $\psi(t)$.

We have introduced the idea of a customer moving from state to state of a Markov process. Now further notation is necessary to specify the condition of the system more precisely. This is required because knowledge of the number of customers in each state of the Markov process does not enable us to decide whether the next customer arrival will be accepted or rejected; this depends on the total number of demands that were placed by these customers.

Let $s$ be the spare stock, and $a_{i j}$ be the customers in the system who have placed an order for $i$ units and who are currently in state $j$ of the Markov process. Then a complete description of the system condition is given by 


$$
a_{11} a_{21} \cdots a_{S 1} a_{12} a_{22} \cdots a_{S n}(\tau)=A(\tau)
$$

which specifies that at time $T$ there are $a_{11}$ customers who ordered one unit and who are in state 1 of the Markov process, $a_{21}$ customers who ordered two units and are in state 1 of the Markov process, etc. Since arrivals are compound Poisson, the probability of a customer arriving and demanding $i$ units during a small time interval, $\Delta t$, is $\lambda \Delta t f(i)+o(\Delta t)^{2}$. As defined in Eq. (A. 1), transitions between a state, $j$, to a different state, $k$, of the Markov process in a small time interval $t$ have probability $q_{j k} \Delta t+o(\Delta t)^{2}$. Thus, in a small time interval, $\Delta t$, the probability of two changes in the system condition will be of order $(\Delta t)^{2}$ and can be neglected.

Note that the Markov process transition probabilities, $q_{j k}$, are independent of $i$, the number of demands per customer. Of course, the $\mathrm{q}_{j k}$ are also independent of the number of customers in the system. Therefore, the $\pi_{j}$ in Eq. (A.2) must express the steady state probability that any customer in the system selected at random is in state $j$ of the Markov process.

Finally we define

$$
\begin{array}{ll}
y=\sum_{i, j} a_{i j} & \text { number of customers in the system; } \\
x=\sum_{i, j} i a_{i j} & \text { number of demands in the system. }
\end{array}
$$

Obviously $0 \leq \mathrm{y} \leq \mathrm{x} \leq \mathrm{s}$, since each customer must demand at least one unit.

Let us describe the ways transitions to and from $\mathrm{A}(T)$ can take place during a small time interval, $\Delta t$. Transitions out of $A(\tau)$ can occur if an arrival demands an amount $s-x$ or less. Otherwise the customer will be turned away as a lost sale. Later we will comment on the alternative policy of allowing partial acceptance of a customer 
order. Transitions out of $A(\tau)$ can also occur if a customer moves from some state, $j$, in the Markov process to another state, $k$, where $j \neq k$.

We can enter $A(\tau)$ by being in a system condition

$$
a_{11} a_{21} \ldots a_{i 1}-1 \ldots a_{s 1} \ldots a_{s n}(\tau),
$$

and having an arrival who requests exactly $i$ units. Another way of entering $A(\tau)$ is by being in a condition

$$
a_{11} a_{21} \ldots a_{s 1} \ldots a_{i j}+1 \ldots a_{s n}(\tau)
$$

and completing service by having a transition to state 1 of the Markov process. For each $i, j$, the probability of this transition ${ }^{*}$ is proportional to the number of customers, $\left(a_{i j}+1\right)$. Finally we may enter $A(\tau)$ by being in a condition

$$
a_{11} a_{21} \ldots a_{i j}+1 \ldots a_{i k}-1 \ldots a_{s n}(\tau)
$$

and having a transition from state $j$ of the Markov process to state $k$ where $j \neq k$ and $k \neq 1$.

Thus the steady state probabilities are given by

$$
\left\{\lambda[f(1)+f(2) \ldots f(s-x)]+\sum_{\substack{j, k \\ j \neq k}} q_{j k} \sum_{i} a_{i j}\right\} p^{a_{11} a_{21}} \ldots a_{s 1} a_{12} \ldots a_{s n}
$$

(A.5) $\quad=\lambda \sum_{i}\left\{\begin{array}{lllll}P_{a_{11} a_{21}} & \ldots & a_{i 1}-1 & \ldots & a_{s 1} \\ \ldots & a_{s n}\end{array}\right) f(i)+$

*This follows from the assumed queue discipline that has one service time drawn from $\psi(t)$ for each customer regardless of his order size. 
(A. 5)

cont.

$$
\sum_{\substack{i, j \\ j \neq 1}}^{\left(a_{i j}+1\right)} q_{j 1} P_{a_{11}} a_{21} \ldots a_{s 1} \ldots a_{i j}+1 \ldots a_{s n}
$$

$$
+\sum_{\substack{i, j, k \\ j \neq k \\ k \neq 1}}\left(a_{i j}+1\right) q_{j k} a a_{11} \ldots a_{i j}+1 \ldots a_{i k}-1 \ldots a_{s n}
$$

The number of equations in this set can be enormous. Let us simplify by adding together all equations with the property that the $\mathrm{P}^{\prime} \mathrm{s}$ on the left-hand side of Eq. (A.5) have the same number of customers, $y$, and the same number of demands, $x$. By construction, this will be the steady state probability, $\varphi_{y, x}$, that there are $y$ customers with a total of $x$ demands. Note the last term on the right-hand side of Eq. (A,5) gives $P^{\prime} S$ with the same values of $y$ and $x$. We shall combine these two terms.

We shall combine the second term on the left-hand side with the rightmost term. In the second term on the left-hand side of Eq. (A.5) we observe that the summation $\sum_{i} a_{i j}$ is the total number of customers in state $j$ of the Markov process. But, the steady state probability that a customer is in state $j$ of the Markov process is $\pi_{j}$ independent of the number of customers, or the demands those customers make. Therefore, given that there are a total of $y$ customers, the expected number of customers in state $j$ of the Markov process is simply $y \pi_{j}$ * The same argument is applied to the last term on the right-hand side of Eq. (A.5). An inductive approach simplifies the algebra. Invoking Eq. (A.3) and subtracting this term from the term discussed above yields,

$$
\left\{\sum_{k=2}^{n} q_{1 k} \pi_{1}+y \sum_{k \neq 2} q_{2 k} \pi_{2} \cdots+y+\sum_{k=1}^{n-1} q_{n k} \pi_{n}\right.
$$

(A.6) $-y \sum_{k=2}^{n} q_{1 k} \pi_{1}-y \sum_{k=3}^{n} q_{2 k} \pi_{2} \ldots .-y \sum_{k=2}^{n-1} q_{n k} \pi_{n}\left\{\varphi_{y, x}=y\left\{\sum_{j=2}^{n} q_{j k} \pi_{j}\right\} \quad \varphi_{y, x}=\frac{y}{T} \varphi_{y, x}\right.$. 
The three remaining terms in $\mathrm{Eq}$. (A.5) simplify readily to transform it to the following form:

$$
\lambda \varphi_{y, x} \sum_{i=1}^{s-x} f(i)+\frac{y_{\varphi}}{T} y, x=
$$

$$
\lambda \sum_{i=1}^{x-y+1} \varphi_{y-1, x-i} f(i)+\frac{(y+1)}{T} \sum_{i=1}^{s-x} \varphi_{y+1, x+i} .
$$

Direct substitution shows that Eq. (A.7) is satisfied by

$$
\varphi_{y, x}=\frac{(\lambda T) y_{e}^{-\lambda T}}{y !} f^{* *}(x)
$$

where $\mathrm{f}^{\mathrm{y}^{*}}(\mathrm{x})$ is the $\mathrm{y}$ fold convolution of the compounding distribution, f. It is the probability that $y$ samples from $f$ yield a sum of $x$. Thus the probability of $x$ units in resupply is simply obtained by summation over $\mathrm{y} \leq \mathrm{x}$ customers and normalization:

$$
\text { (A.9) } h(x)=\frac{\sum_{y=0}^{x} \frac{(\lambda T)^{y} e^{-\lambda T}}{y !} f^{y^{*}}(x)}{\sum_{x=0}^{s} \sum_{y=0}^{x} \frac{(\lambda T)^{y} e^{-\lambda T}}{y !} f^{y *}(x)}=\frac{p(x \mid \lambda T)}{\sum_{w=0}^{s} p(w \mid \lambda T)} \quad 0 \leq x \leq s \text {. }
$$

We have found the stationary probabilities for the case in which the customer.'s complete order is either accepted or rejected, depending on the system availability. As mentioned above, we could have allowed partial fills of a customer order. Under this rule only the first term of Eq. (A. 5) need be modified to show that with $x<s$ inits in resupply any arrival will cause a transition out of $A(\tau)$. The equations for $\varphi_{y, s}$ must be altered, however, to show that there are many additional ways transitions can be made into that state: 


$$
\lambda \varphi_{y, x}+\frac{y}{T} \varphi_{y, x}=\lambda \sum_{i=1}^{x-y+1} \varphi_{y-1, x-i} f(i)+\frac{y+1}{T} \sum_{i=1}^{s-x} \varphi_{y+1, x+1} \quad 0 \leq x<s
$$

(A.10) $\quad \frac{y}{T} \varphi_{y, s}=\lambda \sum_{i=1}^{s-y+1} \varphi_{y-1, s-i} \sum_{m=i}^{\infty} f(m)$

The solution is found by substitution and normalization:

$$
\begin{aligned}
& h(x)= \frac{\sum_{y=0}^{x} \frac{(\lambda T)^{y} e^{-\lambda T}}{y !} y^{*}(x)}{\Delta(s)}=\frac{p(x \mid \lambda T)}{\Delta(s)} \quad 0 s x<s ; \\
& \text { (A.11) } h(s)=\frac{\sum_{y=0}^{s} \frac{(\lambda T)^{y} e^{-\lambda T}}{y !} \sum_{i=s}^{\infty} f^{y^{*}}(i)}{\Delta(s)} ;
\end{aligned}
$$

where

$$
\Delta(s)=\sum_{w=0}^{s-1} p(w \mid \lambda T)+\sum_{y=0}^{s} \frac{(\lambda T)^{y} e^{-\lambda T}}{y !} \sum_{i=s}^{\infty} f^{y *}(i)
$$

We note the curious result that for $0 \leq x<s$, the numerators for $h(x)$ under the partial fill rule are identical with the numerators for $h(x)$ under the complete fill rule. Due to the fact that $h(s)$ is larger under the partial fill rule, the normalization factor in this case is, of course, larger. As $s \rightarrow \infty$, the two probability distributions become identical.

It should be emphasized that these probabilities are easy to compute and that they do not depend on the resupply distribution itself, but only on the mean of the resupply distribution. 


\section{PROOF OF THEOREM 3}

Takacs (5) proved the following theorem: Let demand be Poisson with rate $\lambda$ and the resupply time be an arbitrary distribution $\psi(t)$ with mean $T$. In the backorder case the probability that any unit in resupply in the steady state will still be in the resupply process after a time interval $T$ is given by

$$
\alpha=(1 / T) \int_{\tau}^{\infty}[1-\Omega(t)] d t
$$

where

$$
\Omega(t)=\int_{0}^{t} \psi(\xi) \mathrm{d} \xi
$$

We shall first derive Eq. (13) for Poisson demand. In order to have $y$ customers in resupply at time $t+\tau$, each of whom was in the resupply process at time $t$, there must have been $y$ customers at time $t$ and no resupply completions in the interval $\tau$, or $y+1$ customers at time $t$ and exactly one resupply completion, etc.

$$
\begin{aligned}
\mathrm{P}\left(\begin{array}{c}
\mathrm{y} \text { customers at time } \\
\mathrm{t}+\mathrm{T} \text { each of whom } \\
\text { was in resupply at } \mathrm{t}
\end{array}\right)= & (\lambda \mathrm{T})^{\mathrm{y}} \mathrm{e}^{-\lambda \mathrm{T}} \alpha^{\mathrm{y}} / \mathrm{y} ! \\
& +\left((\lambda \mathrm{T})^{\mathrm{y}+1} \mathrm{e}^{-\lambda \mathrm{T}}(\mathrm{y}+1) \alpha^{\mathrm{y}}(1-\alpha)\right) /
\end{aligned}
$$

$$
\begin{aligned}
& (y+1) !+\ldots \\
= & \left((\lambda T \alpha)^{y} e^{-\lambda T} / y !\right)[1+(\lambda T)(1-\alpha) \\
& \left.+\left((\lambda T)^{2} / 2\right)(1-\alpha)^{2} \ldots\right] \\
= & (\lambda \mathrm{T} \alpha)^{y} e^{-\lambda T \alpha} / y !
\end{aligned}
$$

We have actually proved Theorem 3a. for compound Poisson demand in the back-order case since the distribution of demand per customer, $\left\{f_{i}\right\}$, is independent of the customer generation, i.e., 


$$
P\left(\begin{array}{c}
x \text { demands at time } \\
t+\tau \text { each of which } \\
\text { was in resupply at } t
\end{array}\right)=\sum_{y=0}^{x}\left((\lambda T \alpha)^{y} e^{-\lambda T \alpha} / y !\right) f^{y *}(x)
$$

To complete the proof of theorem 3 for the lost sales case, we shall utilize the proof of theorem 2. We recall from Eq. (A.2) that the steady state probability a customer is in state $j$ of the Markov process $\pi_{j}$, is independent of the number of customers or demands per customer. Therefore, in the steady state any customer in resupply has a fixed probability, denoted by $\beta$, of still being in the resupply process after a time interval $\tau$. This probability is a function only of the $q_{j k}$ matrix which is chosen to approximate the resupply distribution, $\psi(t)$. But theorem 2 with the same $q_{j k}$ matrix is valid for both the lost sales case and the backorder case. Thus $\beta$ in the lost sales case must equal $\alpha$ in the backorder case which is given by Eq. (A.13).

In a derivation analogous to Eq. (A.14), we find for the Poisson lost sales case

$$
\begin{aligned}
P\left(\begin{array}{c}
y \text { customers at } \\
\text { time } t+T \text { each } \\
\text { of whom was in } \\
\text { resupply at } t
\end{array}\right)= & (1 / A)\left[(\lambda T)^{y} e^{-\lambda T} \alpha^{y} / y !\right. \\
& +(\lambda T)^{y+1} e^{-\lambda T}(y+1) \alpha^{y}(1-\alpha) /(y+1) !
\end{aligned}
$$

$$
\begin{aligned}
& \left.+\ldots+(\lambda T)^{s} e^{-\lambda T} s ! \alpha^{y}(1-\alpha) s-y / s !(s-y) ! y !\right] \\
= & (1 / A)\left((\lambda T \alpha)^{y} e^{-\lambda T} / y !\right)[1+\lambda T(1-\alpha) \\
& \left.+\ldots+[\lambda T(1-\alpha)]^{s-y} /(s-y) !\right]
\end{aligned}
$$

where

$$
A=\sum_{x=0}^{S}(\lambda T)^{x} e^{-\lambda T} / x ! \text { for } y \leq s
$$


Note that the probabilities, while easy to compute, are no longer normalized Poisson values.

Finally the same approach for the compound Poisson lost sales case $^{*}$ yields

(A. 18) $\begin{aligned} & P\left(\begin{array}{l}y \text { customers and } x \\ \text { demands at time } t+T \\ \text { each of which was } \\ \text { in resupply at } t\end{array}\right)=\frac{1}{B}\left[\frac{(\lambda T)^{y} e^{-\lambda T} \alpha_{f} y^{* *}(x)}{y !}\right. \\ & +\sum_{k=1}^{s-x} \frac{(\lambda T)^{y+1} e^{-\lambda T}(y+1) ! \alpha^{y}(1-\alpha) f(y+1) *(x+k)}{(y+1) ! y ! 1 !} \frac{f(k) f^{y^{*}}(x)}{f(y+1) *(x+k)}\end{aligned}$

$\left.\ldots+\frac{(\lambda T)^{s-x+y} e^{-\lambda T}(s-x+y) ! \alpha^{y}(1-\alpha)^{s-x}}{(s-x+y) !(s-x) ! y !} \frac{f^{(s-x+y) *}(s) f^{(s-x) *}(s-x) f^{y^{*}}(x)}{f^{(s-x+y) *}(s)}\right]$

$=\frac{1}{B}\left[\frac{(\lambda T \alpha)^{y} e^{-\lambda T}}{y !} f^{y *}(x)\left\{1+\lambda T(1-\alpha) \sum_{k=1}^{s-x} f(k)\right.\right.$

$\left.\left.+\left[\frac{(\lambda T)(1-\alpha)}{2 !}\right]^{2} \sum_{k=2}^{s-x} f^{2 *}(k)+\ldots+\left[\frac{(\lambda T)(1-\alpha)}{(s-x) !}\right]^{s-x}{ }^{(s-x) *}(s-x)\right\}\right]$

where

$$
B=\sum_{x=0}^{s} \sum_{y=0}^{x}\left((\lambda T)^{y} e^{-\lambda T} / y^{\prime}\right) f^{y^{*}}(x) \text { for } y \leq x \leq s
$$

The probability of $x$ demands at $t+T$ each of which was in resupply at $t$ is obtained by suming Eq. (A.18) over $y \leq x$.

\footnotetext{
We consider the complete fill case only. The partial fill case is only slightly more complicated.
} 
Appendix B

COMPUTATION OF STUTTERING POISSON DENSITY FUNCTION AND

MEASURES OF PERFORMANCE IN THE BACKORDER CASE

This appendix describes an algorithm for computing the density function of the stuttering Poisson (geometric Poisson) and the measures of performance for the backorder case described above -ready rate, fills, and services. We derive recursion formulas for each function, and display the IBM FORTRAN II computer program for these calculations. Sample output is shown in Table 1.

Letting the mean resupply time be unity, the compound.Poisson density function is

$$
p(x \mid \lambda)=\sum_{y=0}^{x} \frac{\lambda^{y} e^{-\lambda}}{y !} f^{y *}(x)=\operatorname{pr}\{x \text { demands }\}
$$

where $\mathrm{f}^{\mathrm{y}^{*}}(\mathrm{x})$ is the $\mathrm{y}$ fold convolution of $\left\{\mathrm{f}_{j}\right\}$. In the stuttering Poisson, $\left\{f_{j}\right\}$ is given by

$$
f_{j}=(1-p) \rho^{j-1} \quad j \geq 1,0 \leq p<1 \text {. }
$$

The stuttering Poisson is a two-parameter distribution with mean $X=\lambda /(1-p)$ and variance $V=\lambda(1+p) /(1-p)^{2}$.

To simplify notation let

$$
p(x \mid \lambda)=\sum_{y=0}^{x} \zeta(y) \theta(x-y \mid y)
$$

where

$$
\begin{aligned}
& \zeta(y)=\frac{\lambda^{y} e^{-\lambda}}{y !} \equiv \operatorname{pr}\{y \text { customers }\}, \text { and } \\
& \theta(z \mid y)=f^{y^{*}}(z+y) \equiv \operatorname{pr}\{z+y \text { units demanded } \mid y \text { customers }\} .
\end{aligned}
$$


Then:

$$
\begin{aligned}
\theta(0 \mid 0) & =1 ; \\
\theta(z \mid 1) & =(1-\rho) \rho^{z} ; \\
\theta(z \mid y) & =\sum_{w=0}^{z} \theta(w \mid y-1) \theta(z-w \mid 1)=(1-\rho) \sum_{w=0}^{z-w} \theta(w \mid y-1) ; \\
\theta(z \mid y)-\theta(z-1 \mid y) & =(1-\rho)\left\{\theta(z \mid y-1)+\sum_{w}^{z-w} \theta(w \mid y-1)-\sum \rho^{z-1-w} \theta(w \mid y-1)\right\} \\
& =(1-\rho)\left\{\theta(z \mid y-1)-(1-\rho) \sum_{w=0}^{z} \rho^{z-1-w} \theta(w \mid y-1)\right\} \\
\theta(z \mid y) & =(1-\rho)\{\theta(z \mid y-1)-\theta(z-1 \mid y)\} ;
\end{aligned}
$$

As in Appendix A, we define $\varphi_{y, x}$ to be

$$
\begin{aligned}
\varphi_{y, x} & \equiv p r\{y \text { customers and } x \text { demands }\} \\
& =\zeta(y) \theta(x-y \mid y) \\
& =\zeta(y)[\rho \theta(x-y-1 \mid y)+(1-\rho) \theta(x-y \mid y-1)] \\
& =\rho \zeta(y) \theta(x-y-1 \mid y)+(1-\rho) \frac{\lambda}{y} \zeta(y-1) \theta(x-y \mid y-1) .
\end{aligned}
$$

Thus the basic recursion is

$$
\varphi_{y, x}=\rho \varphi_{y, x-1}+(1-\rho) \frac{\lambda}{y} \varphi_{y-1, x-1},
$$


and the stuttering Poisson density is

$$
p(x \mid \lambda)=\sum_{y=0}^{x} \varphi_{y, x}
$$

Since ready rate is the distribution function, the recursion formula is obviously

$$
R(s)=\sum_{x=0}^{s} p(x \mid \lambda)=R(s-1)+p(s \mid \lambda) .
$$

The recursion formula for the expected number of fills is computed from Eq. (10) by substituting for $\mathrm{f}_{j}$ from Eq. (B.2)

$$
\begin{aligned}
F(s) & =\lambda\left\{R(s-1)+\rho R(s-2)+\rho^{2} R(s-3) \ldots+\rho^{s-1} R(0)\right\} \\
& =\lambda R(s-1)+\rho F(s-1) .
\end{aligned}
$$

The recursion formula for the expected number of units in routine resupply is obtained from Eq. (11):

$$
\begin{aligned}
s(s) & =\sum_{x=1}^{s} x p(x \mid \lambda)+s \sum_{x=s+1}^{\infty} p(x \mid \lambda) \\
& =\sum_{x=1}^{s-1} x p(x \mid \lambda)+p(s \mid \lambda)+(s-1) p(s \mid \lambda) \\
& +(s-1) \sum_{x=s+1}^{\infty} p(x \mid \lambda)+\sum_{x=s+1}^{\infty} p(x \mid \lambda) \\
& =s(s-1)+1-R(s-1) .
\end{aligned}
$$$$
\text { (B.9) }
$$ 
Since the FORTRAN II program to accomplish the above computations is short, we shall describe it here. First we define the following FORTRAN variables:

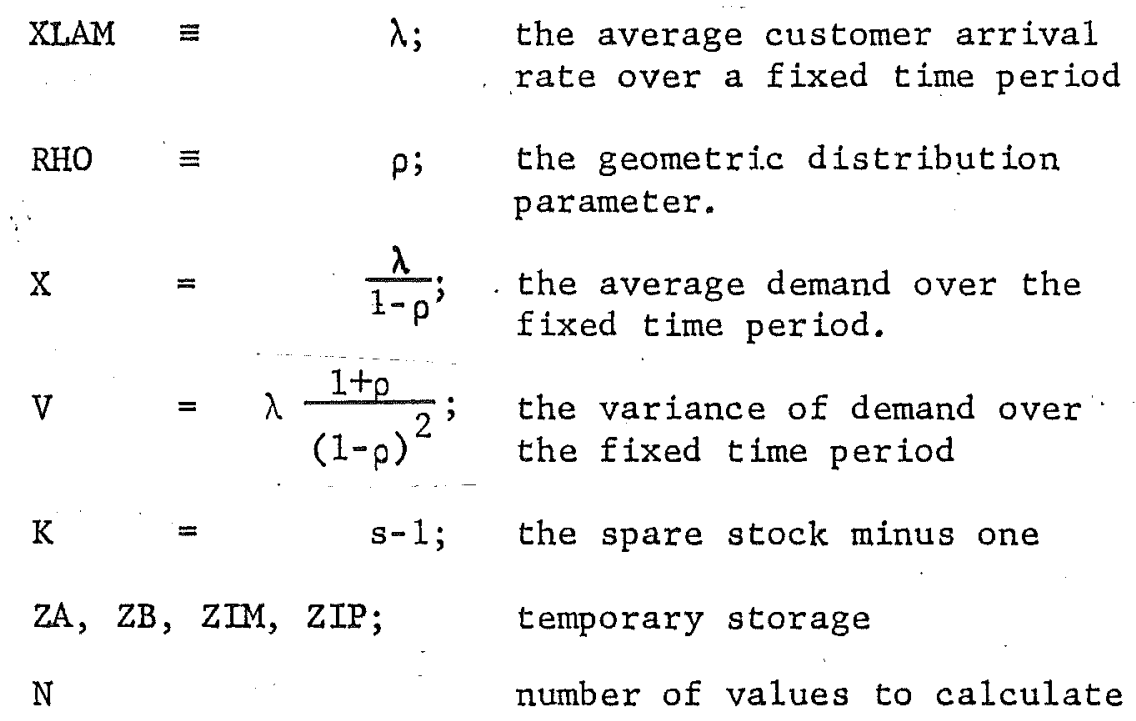


, 


\section{REFERENCES}

1. Aviation Material Management Improvement Program, Final Report, Office Assistant Secretary of Defense (Systems and Logistics), August 17, 1962, Table No. 1.

2. Palm, C., "Analysis of the Erlang Traffic Formula for Busy-Signal Arrangements," Ericsson Technics, No. 5, 1938, pp. 39-58.

3. Hadley, G., and T. M. Whitin, Analys is of Inventory Systems, Prentice-Ha11, Inc., Englewood Cliffs, New Jersey, 1963, pp. 204-212.

4. Galliher, H. P., Philip M. Morse, and M. Simond, "Dynamics of Two Classes of Continuous Review Inventory Systems," Journal of the Operations Research Society of America, Vo1. 7, No. 3, May-June, 1959, pp. 362-384.

5. Taḱacs, L., Introduction to the Theory of Queues, Oxford University Press, New York, 1962, pp. 159-162.

6. Arrow, K J., S. Karlin, and H. Scarf, Studies in the Mathematical. Theory of Inventory and Production, Stanford University Press, Stanford, California, 1958, pp. 304-307.

7. Feller, W., An Introduction to Probability Theory and its App 1ications, Vol. 1, 2nd ed., John Wiley and Sons, Inc., New York, 1950, pp. 268-272. 